\title{
Líneas de defensa y el índice de desempeño en Empresas Estatales Federales Brasileñas*
}

\author{
Defence lines and the performance index in the Brazilian state-owned federal enterprises \\ Linhas de defesa e o índice de desempenho em empresas estatais federais Brasileiras
}

Davi Jônatas Cunha Araújo ${ }^{\text {a }}$

Universidad Federal de Paraiba, UFPB, Brasil

davijonatasss@gmail.com

ORCID: http://orcid.org/0000-0002-7819-5691

Paulo Roberto Nóbrega Cavalcante

Universidad Federal de Paraíba, UFPB, Brasil

ORCID: http://orcid.org/0000-0002-1702-0433

Wenner Glaucio Wenner Glaucio Lopes Lucena

Universidad Federal de Paraiba, UFPB, Brasil

ORCID: http://orcid.org/0000-0002-2476-7383
DOI: https://doi.org/10.11144/Javeriana.cc20-50.ldid

Fecha de recepción: 18 Octubre 2018

Fecha de aprobación: 27 Junio 2019

Fecha de publicación: 30 Noviembre 2019

\section{Resumen:}

En este artículo se analiza si la alta administración, las unidades de control y conformidad, y la auditoría interna -las líneas de defensa (Instituto de los Auditores Internos, 2013) - de las empresas estatales brasileñas, están relacionadas con el desempeño de esas instituciones, a través del Índice de Desempeño de Empresas Estatales (IDESEMPEST). Se empleó la combinación del análisis de contenido y la regresión lineal múltiple, en una investigación documental con los informes de administración, de gestión y de la auditoría interna divulgados en los websites de 24 estatales federales en 2016. Los resultados muestran que la alta administración, la unidad de control y cumplimiento y auditoría interna no tienen una relación significativa con el comportamiento del IDESEMPEST. A pesar de que la alta administración y la auditoría interna funcionan de manera alineada y la unidad de control y conformidad se correlacionan con el desempeño de las estatales, las líneas de defensa no tienen poder explicativo sobre el desempeño obtenido de las empresas estatales.

Códigos JEL: M10, M40, M29.

Palabras clave: desempeño, líneas de defensa, empresas estatales, análisis de contenido.

\begin{abstract}
:
The aim of this study was to verify how high management, control and compliance units and the internal audit of Brazilian stateowned companies, when recognized as lines of defense (Institute of Internal Auditors, 2013), are related to the performance of these organizations, through the Performance Index of State Enterprises (IDESEMPEST). In order to achieve this, the combination of content analysis and multiple linear regression was used in documentary research with the management, management and internal audit reports published on the websites of 24 federal state-owned companies in 2016. The test result Significance of $\beta 1$, which corresponds to the High Administration, did not present a significance relation with the state performance index. The significance tests of $\beta 2$ and $\beta 3$, corresponding to the control and compliance unit and internal audit, also did not show a significance relation with IDESEMPEST. Although high management and internal audit functioned in an aligned way and the control and compliance unit correlated with stated-owned enterprises performance, the lines of defense do not have the power to explain how the performance was obtained in these state-owned companies.
\end{abstract}

JEL Codes: M10, M40, M29.

Keywords: Performance, defence lines, state-owned enterprises, content analysis.

\section{Resumo:}

Este artigo analisa se as unidades da alta administração, controle e conformidade e auditoria interna -às linhas de defesa (Institute of Internal Auditors, 2013) - de empresas estatais brasileiras, estão relacionadas ao desempenho dessas instituições,

Notas de autor:

\footnotetext{
a Autor de correspondencia. Correo electrónico: davijonatasss@gmail.com
} 
através do Índice de Desempenho da Empresa Estatal (IDESEMPEST). A combinação da análise de conteúdo e da regressão linear múltipla foi usada em uma investigação documental com os relatórios de administração, gestão e auditoria interna publicados nos websites de 24 empresas estaduais federais em 2016. Os resultados mostram que a alta administração, a unidade de controle e conformidade e a auditoria interna não apresentam relação de significância com o comportamento do IDESEMPEST. Embora a alta administração e a auditoria interna trabalhem de maneira alinhada e a unidade de controle e conformidade se correlacione com o desempenho das empresas estatais, as linhas de defesa não têm poder explicativo sobre o desempenho obtido pelas empresas estatais.

Códigos JEL: M10, M40, M29.

Palavras-chave: desempenho, linhas de defesa, empresas estatais, análise de conteúdo.

\section{INTRODUCCIÓN}

La eficacia y la eficiencia de las organizaciones se evidencian en su desempeño (Bonacim \& Araújo, 2010), como consecuencia del logro de los objetivos organizacionales (Müller, 2003). El desempeño debe ser asegurado por los criterios de gestión de riesgos y controles establecidos por las unidades de gobierno de las organizaciones (Marks, 2016). Una administración inadecuada de los riesgos de una organización puede impedir el logro de sus objetivos y, por lo tanto, una reducción en el desempeño (Anderson \& Eubanks, 2015).

En la declaración de posicionamiento del Instituto de Auditores Internos -IAI- (2013), el control de la alta administración es la primera línea de defensa en la gestión de riesgos, pues son responsables de establecer los objetivos y procesos que permiten gestionar los riesgos que se puedan presentar en la organización. Las unidades de control interno y supervisión de conformidad representan la segunda línea de defensa, y la auditoría interna representa la tercera. El control de la segunda línea ayuda a monitorear los controles de la alta administración, y la auditoría interna proporciona evaluaciones sobre la eficacia tanto de la alta administración como de los controles internos y de la conformidad (IAI, 2013).

La ejecución del modelo del IAI (2013) en las organizaciones puede diferir de lo que se propone en teoría: se pueden presentan situaciones en las que el interés del gestor en controlar riesgos sea ilegítimo, o el aumento de la complejidad de estos riesgos, que afectan el funcionamiento adecuado de las líneas de defensa y sus consecuencias afectarán el desempeño.

La relación entre la gestión de riesgos y el desempeño financiero ha sido analizada por algunos estudios empíricos. Doyle, Ge y McVay (2007) señalaron que las organizaciones más complejas, que crecen rápidamente o pasan por reestructuraciones, presentan controles más frágiles y por ello muestran un desempeño financiero más débil. Los estudios de Martin, Santos y Dias Filho (2004), DeFond y Zhang (2014) y Kausar, Shroff y White (2016), subsidiaron la relación lineal entre la gestión de riesgos y el desempeñofinanciero por líneas de defensa. Martin et al. (2004) apuntaron que las acciones de la alta administración y de las controladoras están directamente relacionadas con el desempeño financiero de las compañías.

DeFond y Zhang (2014) destacaron la relación entre la auditoría y control interno para gestionar riesgos que afectan el desempeño. Entre mejor sea la auditoría interna y los controles internos estén bien alineados, mejor se gestionarán los riesgos. Kausar et al. (2016) también atribuyeron la función de mejor gestionar riesgos y asegurar el desempeño a la auditoría.

El objetivo de este estudio fue comprobar la relación entre la gestión de riesgos y el desempeño, por líneas de defensa: cuanto mejor sea la gestión de riesgos por la alta administración, control y auditoría interna, mejor el desempeño de las compañías, ya que la propuesta del modelo del IAI (2013) es viabilizar el alcance de los objetivos organizacionales por cada una de las líneas. Además, los hallazgos de los estudios existentes apuntan que la gestión adecuada de riesgos hace que las compañías obtengan mejores resultados financieros.

Algunas empresas estatales brasileñas fueron seleccionadas para estudiar la gestión de riesgos, por estar sometidas a las directrices del Ministerio de Planificación, Desarrollo y Gestión -MPDG- que debe ejecutar las directrices del IAI (2013), y por presentar un desempeño financiero relativamente estable. El presente estudio busca responder la siguiente pregunta: ¿Cómo la alta administración, las unidades de 
control interno y conformidad y la auditoría interna de empresas estatales brasileñas, en tanto líneas de defensa, están relacionadas con el desempeño de esas organizaciones?

Para responder la pregunta de investigación se utilizaron variables proxies: se asigna el valor uno (1) cuando se atendía la función y cero (0) cuando no se atendía, y luego se hizo una suma para cada línea de defensa. La atención a la lista de chequeo fue considerada para mostrar la ejecución de las funciones en los informes de administración y gestión de las estatales.

La división entre el resultado de la suma y la cantidad de funciones establecidas por el IAI (2013) para la alta administración (3), unidad de control y cumplimiento (9) y auditoría interna (3) fue posteriormente efectuada, para establecer las proxies como índices para cada línea de defensa.

La proxy de desempeño fue definida por el Índice de Desempeño de las estadísticas (IDESEMPEST), construido por la media ponderada entre los valores de los indicadores financieros y no financieros divulgados en los informes de administración y gestión de las estatales. Se utilizó una regresión lineal para probar la relación entre las líneas de defensa y el desempeño. Cada índice de las líneas representa una variable independiente y la variable dependiente es el desempeño representado mediante el IDESEMPEST.

Se hizo el análisis de contenido por medio de software para comparar los resultados con los del análisis de la regresión econométrica. Para el análisis de contenido se consideró la clasificación jerárquica descendente de las palabras por intención de sentido, nivel de significancia y el contexto en que las palabras fueron utilizadas, en el intento de percibir alguna relación entre las líneas y el desempeño, por otras vías.

El artículo está estructurado en cinco secciones que siguen a esta introducción. La segunda presenta la fundamentación teórica, que relaciona la literatura de la gestión y control de riesgos con el desempeño, por líneas de defensa. La tercera sección presenta los procedimientos metodológicos de la investigación. La cuarta y quinta sección evidencian, respectivamente, el análisis de los resultados y las consideraciones finales.

\section{FUNDAMENTACIÓN TEÓRICA}

Al proponer el modelo de las tres líneas de defensa, el Instituto de Auditores Internos -IAI- (2013) presentó un modelo para que los directivos de cualquier organización gestionen los riesgos que se pudieran llegar a presentar. El objetivo de este modelo fue aclarar las funciones y las responsabilidades de la alta administración, de las unidades de control interno y de conformidad, y de la auditoría interna en la gestión de riesgos.

Los positions papers de Doughty (2011), Tabuena (2015), Clemens (2014), Mont (2015) y Marks (2016) se publicaron con el objetivo de explicar cómo se podría aplicar el modelo de las tres líneas de defensa en las organizaciones. Los demás documentos se publicaron con el fin de aclarar el funcionamiento de cada línea (KPMG, 2016), y el análisis conjunto de las tres líneas asociadas a otros modelos conceptuales (Seago, 2015). Para el caso de Brasil, Porta (2011) y Linczuk (2012) analizan la actuación de la auditoría interna en el ámbito de la gobernanza. El estudio de Quadros (2015) fue un poco más allá al analizar la relación entre las funciones de la auditoría interna y el desempeño de las organizaciones.

El propósito del modelo de las tres líneas es ayudar a resolver los conflictos que impiden el logro de los objetivos organizacionales y que perjudican el desempeño de las organizaciones (Anderson \& Eubanks, 2015). En este sentido, los position papers de Tabuena (2015) y del Banco de Pagos Internacionales (BIS, 2012) evidenciaron las directrices para facilitar la implementación del modelo del IAI (2013). Dichos papers recomendaron la implementación de este modelo de gestión de riesgo a la luz del desempeño de las compañías.

De acuerdo con el planteamiento del IAI (2013), el modelo de las tres líneas funciona de manera adecuada en cualquier organización independientemente de su tamaño o complejidad. Las empresas estatales federales brasileñas deben tener unidades responsables para la gestión de riesgos, en cumplimiento de las directrices de la Comisión Interministerial de Gobierno Corporativo y de Administración de Participaciones Societarias de la Unión -CGPAR-. De las medidas deliberadas por la CGPAR, la que favoreció la implementación de 
las tres líneas en las estatales, fue la atribución de las responsabilidades de la gestión de riesgos a tres unidades de gobierno: alta administración, unidades de control y conformidad y auditoría interna.

Además de establecer responsabilidades en la gestión de riesgos para la alta administración, la auditoría interna y la unidad de gestión de riesgos y conformidad, la CGPAR determinó la existencia de diez instancias mínimas obligatorias de gobernanza para las estatales (MPDG, 2016).

El proceso de gestión de riesgo de las empresas estatales implica factores de alta complejidad. Además, el desempeño financiero de estas empresas no presenta variaciones significativas, en comparación con el desempeño de las empresas de los demás sectores, según lo divulgado por el Ministerio de Planificación, Desarrollo y Gestión (MPDG), en la encuesta de levantamiento de perfil de las estatales en 2016.

El estudio de Kaplan y Norton (1997) muestra que la medición de la evaluación del desempeño de una organización debe considerar indicadores financieros y no financieros, pues los financieros funcionan como una síntesis final del desempeño gerencial y organizacional, mientras que los no financieros posibilitan medir el desempeño de otras áreas: la rentabilidad de clientes, procesos internos, los empleados, etc. No obstante, en un estudio posterior, Luke, Barraket y Eversole (2013) encontraron que las autoridades públicas, al medir su desempeño, tienden a enfatizar más en las medidas financieras que en las no financieras.

El uso conjunto de indicadores financieros y no financieros permite medir el desempeño global de las organizaciones. Si las estatales privilegian las medidas financieras sobre las no financieras para medir su desempeño, los resultados obtenidos no reflejarán el desempeño global; esto puede provenir de un mal funcionamiento de la gestión de riesgos por parte de las líneas de defensa en dichas organizaciones.

El Ministerio de Planificación, Desarrollo y Gestión (2017) recomendó la implementación del modelo de las Tres Líneas de Defensa del IAI (2013), con el apoyo del Comité de Gestión Estratégica de Política de Gestión de la Integridad, Riesgos y Controles Internos. La alta administración representa la primera línea de defensa del modelo del IAI (2013) y debe establecer las funciones para reducir el riesgo de acciones intencionales, gestionar riesgos e implementar acciones correctivas para resolver deficiencias en procesos y controles, para que estén alineados con los objetivos y, en últimas, se asegure el buen desempeño. Se tiene entonces, la primera hipótesis de investigación (H1): existe una relación lineal positiva entre la alta administración y el desempeño de las estatales.

La segunda línea de defensa está ocupada por el organismo cuya responsabilidad comprende un conjunto de funciones amplias de soporte de información, control interno, planificación tributaria, elaboración del presupuesto, medidas operacionales y formulación de estrategias (Lunkes, Gasparetto \& Schnorrenberger, 2011). En este sentido, se formula la segunda hipótesis de la investigación (H2): existe una relación lineal positiva entre la unidad de control y conformidad y el desempeño de las estatales.

La auditoría interna representa la tercera línea del modelo, que conforme a la KPMG (2016), busca mejorar la identificación del riesgo, dar un abordaje prospectivo a las evaluaciones internas, y monitorear la efectividad de la actuación de la alta administración y las unidades de control y cumplimiento. Esta línea de defensa integra los procesos en todas las áreas de riesgo manejables (Clemens, 2014). En las estatales, la auditoría interna es responsable de las evaluaciones exhaustivas e independientes sobre la eficacia de la gestión de riesgos por la alta administración y por las unidades de control y conformidad, además de monitorear el funcionamiento adecuado de esas unidades. Con lo anterior, se formula la tercera hipótesis de la investigación (H3): existe una relación lineal positiva entre la auditoría interna y el desempeño de las estatales.

\section{MÉTODO}

Para probar las hipótesis se empleó la combinación de la investigación cualitativa y cuantitativa como forma de abordaje, teniendo como base una investigación documental realizada en los informes de administración y de 
gestión y en los informes anuales de actividades de la auditoría interna divulgados por la alta administración, unidad de control y conformidad y auditoría interna de las estatales.

Se llevó a cabo un análisis multivariado por medio de regresión lineal múltiple para verificar si las líneas de defensa categorizadas explican el Índice de Desempeño de las Empresas Estatales (IDESEMPEST). Además, se hizo un análisis de contenido para verificar cómo las líneas de defensa sirvieron para proporcionar información en los reportes.

Inicialmente, las empresas estatales seleccionadas para el estudio fueron las 78 empresas indicadas en la encuesta "Perfil de las empresas estatales federales" publicada en el año 2016 por el MPDG. Estas 78 empresas fueron clasificadas como del sector productivo y financiero por el propio MPDG. En el sector productivo participaron las empresas de producción de petróleo y derivados, generación y transmisión de energía eléctrica. En el sector financiero participaron las instituciones que actúan en el Sistema Financiero Nacional, sujetas a las normas y control del Banco Central de Brasil.

La justificación para la selección de las empresas del sector financiero y productivo también se dio por el enfoque conceptual del modelo del IAI (2013): funcionar de manera adecuada, independiente de la complejidad o tamaño de la organización y por el contexto en que esas empresas están insertas en el mercado.

De las 78 empresas inicialmente seleccionadas se filtraron las que no publicaban los informes de la alta administración, de la unidad de control y conformidad y de la auditoría interna en sus sitios institucionales, lo que ocasionó la reducción de la muestra a 29 empresas. Se verificó que cinco de esas empresas no divulgaron indicadores en ninguna de las dimensiones, lo que inviabilizó su continuación en el modelo, y quedaron 24 estatales en la investigación.

\section{Herramientas y procedimiento}

El índice de desempeño de las estatales (IDESEMPEST) fue construido con la media ponderada de los valores de los indicadores financieros y no financieros evidenciados en los informes de la alta administración, de gestión y de la auditoría interna de cada empresa de la muestra en 2016. Estos indicadores fueron segregados entre las dimensiones financiera y no financiera, según se divulga en los informes, totalizando 61 indicadores financieros y 59 no financieros. De los 61 indicadores financieros, los más utilizados fueron el retorno sobre los activos, el endeudamiento a corto y a largo plazo, el margen neto y el porcentaje de ejecución de las inversiones. De los 59 indicadores no financieros, los más utilizados fueron la calidad operativa, el índice de frecuencia y la satisfacción del cliente.

Los indicadores fueron seleccionados cuando las empresas afirmaban que los utilizaban para evaluar el alcance de los objetivos estratégicos y para asegurar el desempeño. Los valores de esos indicadores que se encontraban en porcentajes se convirtieron en números decimales a partir de la división de los valores brutos por 100 para ser incluidos en el modelo de regresión lineal.

La asignación de la misma ponderación $(0,5)$ para la dimensión financiera y para la no financiera responde al equilibrio en la medición del desempeño global de las organizaciones del enfoque de Kaplan y Norton (1997), y que han seguido los estudios que miden el desempeño de las organizaciones como un todo (Luke, Barraket \& Eversole, 2013).

Para calcular el índice de desempeño de cada dimensión se dividieron proporcionalmente las ponderaciones de los aspectos financieros $(0,5)$ y los no financieros $(0,5)$ por la cantidad de indicadores divulgados en cada dimensión, ver la ecuación (1).

$$
\frac{I 1 \times P 1+I 2 \times P 2+\cdots+\ln \times P n}{P 1+P 2+\cdots+P n}=I D D F I N / I D D N F I N
$$


Donde:

$I I=$ Valor del indicador uno

$P 1=$ Peso asignado al indicador uno

$I 2=$ Valor del indicador dos

$P 2=$ Peso asignado al indicador dos

In $=$ Valor del indicador $\mathrm{n}$

$P n=$ Peso asignado al indicador $\mathrm{n}$

$I D D F I N=$ Índice de Desempeño de la Dimensión Financiera

IDDNFIN = Índice de Desempeño de la Dimensión No Financiera

Después de calcular el índice de desempeño por dimensión financiera y no financiera, se efectuó la suma de las medias ponderadas de los valores de esos índices, para calcular el índice de desempeño global por empresa estatal, conforme la ecuación (2):

$$
(I D D F I N \times 0,5)+(I D D N F I N \times 0,5)=I D E S E M P E S T
$$

El índice de desempeño de las estatales (IDESEMPEST) es la variable dependiente (Y) de la regresión lineal, después del cálculo de su logaritmo. Se aplicó el logaritmo natural para estandarizar el rango numérico de los índices de desempeño global, conforme al enfoque de Girão (2016). El cálculo del logaritmo viabilizó la investigación, pues aseguró la permanencia de las estatales en la muestra, después de normalizar los outliers existentes en el intervalo de los datos. Al logaritmo del índice de cada estatal se sumó uno (1), para asegurar que las empresas que no informaron indicadores en la misma proporción en las dimensiones financieras y no financieras se mantuvieran en la muestra, dado que, el logaritmo natural no considera valores iguales a o por debajo de cero en el intervalo de datos.

La interpretación para los valores obtenidos por IDESEMPEST es cuanto mayor es su valor, más proporcional es la medición de desempeño en las estatales. Cuanto menor es el valor del IDESEMPEST, menos proporcional fue el uso de indicadores financieros y no financieros para medir su desempeño global. En ese caso, las líneas de defensa consideraron otro grupo de indicadores cuando midieron el desempeño global de la organización.

Las variables independientes se clasificaron a través de índices de atención a las directrices recomendadas por el IAI (2013) en su declaración de posicionamiento, para la alta administración, unidad de control y conformidad y para auditoría interna.

La construcción de estos índices se hizo inicialmente asignando el número uno (1) a las directrices que cumplen la lista de verificación de IAI (2013) para cada línea de defensa y cero (0) cuando no cumplían las directrices. Después del levantamiento de las directrices atendidas por líneas, se efectuó su suma y la consiguiente división del valor resultante por la cantidad de directrices recomendadas por el listado de chequeo para cada línea en la declaración de posicionamiento.

Las directrices del modelo de IAI (2013) de la alta administración son las siguientes: la existencia de las funciones que gestionan y tienen la propiedad de riesgos (1), si supervisan el riesgo (2), y proporcionan evaluaciones independientes (3). Para la unidad de control si había políticas de gestión de apoyo, definición de roles, responsabilidades y fijación de objetivos para la implementación de estas funciones (1), y si se proporcionaron las estructuras de gestión de riesgos (2). Si se identificaron las cuestiones actuales y emergentes (3), si se hicieron cambios en la tendencia al riesgo implícito de la organización (4), y si existía auxilio a la gerencia para desarrollar procesos y controles de gestión de riesgos (5). Además de estas, también se investigó si existían orientaciones y entrenamiento sobre procesos de gestión de riesgos para el personal (6) y si la alta administración hizo monitoreo e implementación de prácticas eficaces de gestión de riesgos (7). 
En la segunda línea, se investigó si se habían efectuado mecanismos de alerta a la alta administración sobre cuestiones emergentes y cambios en el escenario regulatorio y de riesgos (8) y si existía un monitoreo adecuado y eficaz del control interno, de la precisión y de la integridad del reporte de conformidad con las leyes y reglamentos y la resolución oportuna de deficiencias (9).

En cuanto a las directrices del IAI (2013) para la auditoría interna, se investigó si las prácticas de auditoría interna, de acuerdo con los estándares internacionales reconocidos para esa unidad se llevaron a cabo (1) si había una línea de reporte a un nivel lo suficientemente alto en la organización, de modo que sus responsabilidades se cumplieran de manera independiente (2) y si tenían una línea de reporte activa y eficaz para los órganos de gobernanza (3).

El análisis de los datos se hizo con la estadística descriptiva de la distribución de frecuencia del IDESEMPEST de cada estatal y de los índices correspondientes a las tres líneas de defensa. El análisis descriptivo englobó las medidas de tendencia, dispersión, asimetría y curtosis, para demostrar la viabilidad de las proxies en el modelo de regresión lineal.

Además del análisis de estas medidas, se hizo un análisis de la correlación de Spearman entre el IDESEMPEST y los índices de las líneas de defensa para constatar el nivel de correlación entre esas variables aspirando garantizar la permanencia de ellas en el modelo. Entonces, un primer momento del análisis de los datos corresponde a la correlación de las variables, y un segundo momento, a la regresión lineal múltiple. La ecuación (3) ilustra la relación entre IDESEMPEST, la alta administración, la unidad de control y conformidad y la auditoría interna, reconocidas como líneas de defensa:

$$
\text { LogIDESEMPEST }+1=\beta_{0}+\beta_{1 \text { AltaAdm } .}+\beta_{2 \text { Cont.y Conf } .}+\beta_{3 \text { Audit.Interna }}+\mu
$$

Donde:

Log IDESEMPEST +1 = Logaritmo natural del $\Sigma$ de los promedios ponderados de los índices de desempeño por dimensión financiera (IDDFIN) y no financiera (IDDNFIN)

$\beta O=$ constante

$\beta 1$ AltaAdm.t $_{\cdot t}=$ Índice de atención a las directrices por la alta administración (1 $1^{\mathrm{a}}$ línea)

$\beta 2$ Cont. $y$ Conf $_{\cdot t}=$ Índice de atención a las directrices por la unidad de control y conformidad ( $2^{\mathrm{a}}$ línea $)$

$\beta 3$ Audit. Interna $a_{t}=$ Índice de atención a las directrices por la auditoría interna ( $3^{\mathrm{a}}$ línea)

$\mu=$ Residuos

El análisis multivariado sirvió para probar la relación conceptual entre la alta administración, las unidades de control y conformidad y auditoría interna de las estatales, como líneas de defensa y el IDESEMPEST, como representación del desempeño global de las estatales.

Los cinco supuestos del modelo de mínimos cuadrados ordinarios de la ecuación (1) también se probaron para verificar si se atendieron todos los supuestos y si los resultados estimados eran válidos y posibles para hacer inferencias y asegurar los resultados obtenidos en la investigación.

Las pruebas de Jarque-Bera, Breusch-Pagan y White se efectuaron para probar la normalidad y la homocedasticidad de los datos y de los residuos. Para comprobar la ausencia de multicolinealidad se utilizó la prueba Variance Inflation Factor (VIF). Se realizaron estas pruebas para confirmar la viabilidad del uso de la técnica de regresión lineal en el estudio y asegurar los resultados obtenidos por la aplicación de la técnica.

Se hizo un análisis de contenido con el Iramuteq para comparar los resultados del análisis cuantitativo con el análisis cualitativo. La justificación para la ejecución de este tipo de análisis fue asegurar el rigor de la investigación, a partir de la comparación entre los resultados obtenidos por dos enfoques diferentes de investigación. 
El análisis factorial del contenido de los informes emitidos por la alta administración, unidad de control y conformidad y auditoría interna fue realizó para comparar la forma en que se divulgó el contenido en los informes publicados por esas unidades: administración, gestión y auditoría interna.

Se utilizó la clasificación jerárquica descendente de las palabras por intención de sentido, nivel de significancia y el contexto en que se utilizaron las palabras, para percibir la relación entre los contextos del contenido divulgado en los informes de la alta administración, de las unidades de control y conformidad y de la auditoría interna.

\section{RESULTADOS Y DISCUSIÓN}

El análisis de las medidas de tendencia, dispersión, las pruebas de asimetría y curtosis se presentan en la tabla 1 , en la cual se muestra una media de 0,82 , con una desviación estándar de 0,9208 , de las empresas estatales federales brasileñas que utilizaron indicadores financieros y no financieros para evaluar su desempeño.

La distancia entre el valor máximo y mínimo de IDESEMPEST mostró que la alta administración, la unidad de control y cumplimiento y la auditoría interna presentaron comportamientos distintos en cuanto a la forma en que las estatales utilizaron los indicadores financieros y no financieros para medir su desempeño.

TABLA 1

Estadísticas descriptivas con datos de los informes de administración, de gestión y anuales de la auditoría interna de los websites institucionales de 24 estatales en 2016

\begin{tabular}{lccccccc}
\hline & Promedio & $\begin{array}{c}\text { Desviación } \\
\text { estándar }\end{array}$ & Minimo & Máximo & Asimetria & Curtosis & Valor P \\
\hline IDESEMPEST & 0,8200 & 0,9208 & $-1,4621$ & 2,8260 & 0,8383 & 0,3247 & 0,5798 \\
Alta administración & 0,7917 & 0,2917 & 0,3333 & 1,0000 & 0,0711 & 0,0700 & $0,0483^{* *}$ \\
$\begin{array}{l}\text { Controles y } \\
\text { conformidad }\end{array}$ & 0,8379 & 0,9255 & 0,6667 & 1,0000 & 0,3876 & 0,9287 & 0,6697 \\
Auditoría interna & 0,7638 & 0,2080 & 0,3333 & 1,0000 & 0,5319 & 0,6663 & 0,7392 \\
\hline
\end{tabular}

\footnotetext{
Nota: ${ }^{*}$ significativo al $10 \%,{ }^{* *}$ significativo al $5 \% ;{ }^{* * *}$ significativo al $1 \%$

Fuente: elaboración propia.
}

Los índices de atención a las directrices del IAI (2013) por las tres líneas, presentaron valores medios cercanos: 0,7917, para la alta administración, 0,8379 para las unidades de control y conformidad y 0,7638, para la auditoría interna. Al tratarse de la atención a las directrices recomendadas por el IAI (2013), la alta administración, la unidad de control y conformidad y la auditoría interna de las estatales presentaron un comportamiento similar, diferente del comportamiento presentado por esas líneas cuando miden el desempeño.

El impacto de la diferencia de comportamiento de las tres líneas de defensa en las estatales revela que la alta administración, la unidad de control y conformidad y la auditoría interna, en esas empresas, cumplen las normas establecidas por la gobernanza de cada una, pero la ejecución de esos procedimientos no contribuye a que estas empresas alcancen un mejor rendimiento.

Las directrices más atendidas por las estatales fueron las de las unidades de control y conformidad $(0,8379)$, de forma que evidenciaron más funciones de riesgo, controles internos y conformidad en los informes de gestión, a pesar de la desviación estándar de 0,9255. Esto significa que estas organizaciones cumplen las directrices del IAI (2013) para las unidades de control interno y conformidad, pero no de manera estandarizada, lo que va en contra de lo establecido por el MPDG (2016) para las estatales.

Los índices de atención a las directrices por la alta administración y por la auditoría interna presentaron valores aproximados. Esto implica que la alta administración de las estatales atiende en la misma medida las recomendaciones del IAI (2013) y la auditoría interna. 
Las pruebas de asimetría y curtosis de IDESEMPEST y de los índices de las tres líneas, se efectuaron para constatar si las variables del estudio presentaban distribución normal. El IDESEMPEST presentó asimetría de 0,8383 y curtosis de 0,3247 , con p-valor de 0,5798 . En cuanto al límite de significancia de 0,05 para el p-valor, el índice de desempeño de las estatales presentó distribución normal, sin exceso de curtosis, lo que confirma la aproximación de los valores de todos los índices de desempeño por estatal.

Las estatales presentaron un perfil parecido en el uso de las medidas financieras y no financieras para evaluar su desempeño, independientemente del sector en que actúan y de la actuación de las líneas de defensa. Esto demuestra el hecho de que estas organizaciones pueden incluso parecerse en el proceder de la alta administración, la unidad de control y cumplimiento y la auditoría interna, pero difieren en la forma de medición del desempeño. Además de la confirmación de las pruebas de asimetría y curtosis, esta interpretación se ve confirmada por la aproximación entre los valores máximos $(2,8260)$ y mínimos $(-1,4621)$ del IDESEMPEST.

La alta administración presentó en asimetría de 0,0711 y curtosis de 0,07 en las prueba de las líneas de defensa, considerando el p-valor de 0,0483. Este resultado confirmó cierto nivel de asimetría y cortos en el cumplimiento de las directrices del IAI (2013) para la alta administración de las estatales, lo que indica que la alta administración de las estatales no sigue todas las directrices del IAI (2013) para la primera línea de defensa y tampoco presentó un patrón de procedimientos para la gestión de riesgos por la alta administración.

Este resultado es contrario a lo establecido por el MPDG (2016) sobre la gestión integrada que debe ser mantenida por la alta administración para garantizar el funcionamiento adecuado de la primera línea de defensa. A diferencia del resultado del índice de la alta administración, las pruebas de asimetría y curtosis de la unidad de control y conformidad y auditoría interna, presentaron asimetrías de 0,3876 y 0,5319 , con cortos de 0,9287 y 0,6663 , respectivamente.

Ambos resultados señalan que la unidad de control y cumplimiento y auditoría interna han cumplido con las directrices del IAI (2013). La ausencia de asimetría y del exceso de curtosis en el intervalo de los valores de los índices de las líneas, señala un funcionamiento desproporcionado entre la alta administración, la unidad de control y conformidad y la auditoría interna.

La comparación entre los resultados obtenidos por las diferentes medidas de tendencia y dispersión también mostró que la unidad de control y conformidad de las estatales atiende más las directrices del IAI (2013) que la auditoría interna.

Después del análisis descriptivo, el análisis de la correlación de Spearman evidencia los niveles de correlación entre el IDESEMPEST por cada línea de defensa. La tabla 2 presenta la correlación entre la alta administración, la unidad de control y conformidad y la auditoría interna, como variables explicativas, para verificar la interacción entre estas tres líneas y el desempeño de las empresas estatales.

TABLA 2

Matriz de correlación de Spearman, con datos de los informes de administración, de gestión y anuales de auditoría interna de los websites institucionales de 24 estatales en 2016

\begin{tabular}{lcccc}
\hline & IDESEMPEST & $\begin{array}{c}\text { Alta } \\
\text { administración }\end{array}$ & $\begin{array}{c}\text { Controles y } \\
\text { conformidad }\end{array}$ & $\begin{array}{c}\text { Auditoria } \\
\text { Interna }\end{array}$ \\
\hline IDESEMPEST & 1,0000 & & & \\
Alta administración & 0,1877 & 1,0000 & & \\
& $(0,3798)$ & 0,0671 & & \\
Controles y conformidad & $0,4440^{* *}$ & $(0,7555)$ & 1,0000 & \\
\multirow{2}{*}{ Auditoria interna } & $(0,0298)$ & $0,5869^{*}$ & 0,2683 & \multirow{2}{*}{1,0000} \\
\hline & 0,2713 & $(0,0026)$ & $(0,2049)$ & \\
\hline
\end{tabular}

Nota: ${ }^{*}$ significativo al $10 \%,{ }^{* *}$ significativo al $5 \%,{ }^{* * *}$ significativo al $1 \%$ Fuente: elaboración propia

Como se aprecia en la tabla 2 , las funciones de la alta administración $(0,1877)$ y de la auditoría interna $(0,2713)$ no presentaron correlación con el índice de desempeño, a diferencia de lo que sucede con la unidad 
de control y conformidad (0,4440). Esto significa que las funciones de control interno y cumplimiento influyen más en el desempeño de las estatales que la alta administración y la auditoría interna. Este resultado llama la atención por el hecho de que la unidad de controles internos y de conformidad pareció tener mayor responsabilidad sobre la salvaguardia del desempeño que la administración y la auditoría interna.

La tabla 2 muestra que el índice de las unidades de control y conformidad no presentó correlación con los índices de la alta administración $(0,0671)$ y de la auditoría interna $(0,2683)$. En cuanto a la interacción entre estas líneas, el MPDG (2016) recomienda que las organizaciones identifiquen los riesgos que pudieran surgir y gestionarlos con controles adecuados, de modo que no se perjudiquen los objetivos estratégicos.

Este resultado implica el hecho de que la unidad de control y conformidad de las estatales, además de tener mayor responsabilidad sobre asegurar la medición de desempeño en esas empresas -lo que no debería suceder- funcionan de manera independiente, sin relacionarse con la alta administración y auditoría interna de las estatales. Este resultado va en contra de lo establecido por el IAI (2013), cuando instauró las directrices para el funcionamiento integrado del modelo entre las tres líneas para gestionar riesgos.

El análisis de la correlación entre el IDESEMPEST y las líneas de defensa mostró que la gestión de riesgos no ocurre de manera proporcional entre esas líneas, ya que las funciones de la alta administración y de la auditoría interna estuvieron más relacionadas entre sí, y menos relacionadas con las funciones de la administración unidad de control y conformidad. Esto recae sobre la manera en que se mide el rendimiento en estas organizaciones, pues el índice de la segunda línea presentó más relación con el rendimiento, que la primera y tercera línea.

Para comprobar la relación estadística de significancia entre el IDESEMPEST y los índices de la alta administración, de la unidad de control y conformidad y de la auditoría interna, el análisis con regresión lineal múltiple consideró el IDESEMPEST como variable dependiente y las tres líneas de defensa como variables explicativas; los resultados se presentan en la tabla 3.

TABLA 3

IDESEMPEST y las líneas de defensa con datos de los informes de administración, de gestión y anuales de auditoría interna de los sitios web institucionales de las 24 estatales en 2016

\begin{tabular}{|c|c|c|c|c|}
\hline IDESEMPEST & Coeficiente & $\begin{array}{c}\text { Desviación } \\
\text { estándar }\end{array}$ & t & $\mathbf{P}>|t|$ \\
\hline$\beta_{0}$ & $-3,1926$ & 1,6907 & $-1,89$ & 0,074 \\
\hline$\beta_{1}$ Alta Administración & 0,3152 & 0,7707 & 0,41 & 0,687 \\
\hline$\beta_{2}$ Controles y conformidad & 4,0759 & 2,0422 & 2,00 & $0,060^{\circ}$ \\
\hline \multirow[t]{2}{*}{$\beta_{3}$ Auditoria interna } & 0,4550 & 1,1196 & 0,41 & 0,689 \\
\hline & Prob $>F=0,1503$ & $\mathrm{R}^{2}=0,2285$ & $\mu=15,04$ & \\
\hline $\mathrm{R}^{2}$ ajustado & 0,1128 & VIF (media) & 1,43 & \\
\hline Test $F(3,20)$ & $0,1503 * *$ & White & $0,6064^{* *}$ & \\
\hline Jarque-Bera & $0,1215^{* *}$ & Breusch-Pagan & $0,9538^{* *}$ & \\
\hline
\end{tabular}

Nota: ${ }^{*}$ significativo al $10 \%,{ }^{* *}$ significativo al $5 \%,{ }^{* * *}$ significativo al $1 \%$ Fuente: elaboración propia

La tabla 3 presenta los resultados de la regresión lineal múltiple para probar la relación entre los índices de la alta administración, la unidad de control y conformidad, la auditoría interna y el desempeño. El $\mathrm{R}^{2}$ de 0,2285 evidencia que las funciones de las líneas explican en aproximadamente el $23 \%$ las variaciones ocurridas en el índice de desempeño de las estatales. Es decir, que la alta administración, la unidad de control y conformidad y auditoría interna de las estatales poseen bajo poder de explicación sobre esta forma de medir el desempeño de las estatales, tal vez esto justifique la disparidad en el uso de las medidas financieras y no financieras utilizadas para medir su desempeño.

El valor del $\mathrm{R}^{2}$ ajustado se diferenció del $\mathrm{R}^{2}$ no ajustado en 0,1128 , lo que implica decir que si hubiera la inclusión de otras líneas de defensa en el modelo más allá de las tres ya existentes, las variables explicativas y la constante $\left(\beta_{0}\right)$ reducirían su poder de explicación sobre las variaciones en el IDESEMPEST para el 12\%, lo 
que confirma el hecho de que las líneas de defensa no contribuyeron de manera efectiva al logro de un mejor desempeño en las estatales.

A pesar del resultado señalado por el $\mathrm{R}^{2}$ y $\mathrm{R}^{2}$ ajustado, de que las líneas de defensa y el índice de desempeño de las estatales tienen baja relación, cuando se analizaron individualmente los parámetros de la prueba $\mathrm{F}$ (Prob $>\mathrm{F}=0,1503$ ) los resultados señalaron que la alta administración, la unidad de control y conformidad y la auditoría interna, también presentaron bajo poder de explicación sobre la forma en que se mide el desempeño en las organizaciones.

Este resultado confirma que una línea de defensa por sí sola no explica el desempeño de la organización, lo cual ratifica el enfoque del propio IAI (2013), de Porta (2011), Linczuk (2012) y Quadros (2015), sobre la validez de la actuación conjunta de las líneas de aseguramiento en el modelo conceptual de las tres líneas para el logro de los objetivos en las metas organizaciones y, por consiguiente, un mejor rendimiento.

Para comprobar si se cumplieron todos los supuestos del estimador de los mínimos cuadrados ordinarios, la prueba de la normalidad de Jarque-Bera $(0,1215)$ comprobó la normalidad de la distribución de los residuos de la regresión y la prueba de Breusch-Pagan $(0,9538)$ comprobó que esta distribución es homocedástica, lo que se ratificó a través de la prueba de White $(0,6064)$ garantizando la validez de los resultados estimados. Además, la prueba de las estadísticas Variance Inflation Factor (VIF $=1,43$ ) reveló ausencia de multicolinealidad entre los coeficientes de los índices, lo que garantiza su viabilidad para hacer inferencias.

En cuanto a la prueba de significancia individual de $\beta_{0}(\mathrm{P}>\mathrm{t}: 0,074)$, se verificó que la constante del modelo no fue significativa, lo que apunta al hecho de que el $\beta 0$ (constante), por sí solo tampoco es suficiente para explicar el IDESEMPEST, demandado la existencia de otras variables para explicar las variaciones en el desempeño de las estatales.

El resultado de la prueba de significancia del $\beta 1$ (P> t: 0,687), que corresponde a Alta Administración, no presentó relación de significancia con el índice de desempeño de las estatales, de manera que se rechaza la hipótesis H1. Este resultado controvierte los conceptos establecidos por el propio IAI (2013), así como lo esperado por el MPDG (2016), pues al establecer la implementación del modelo de las tres líneas, la alta administración es la responsable de definir los objetivos estratégicos y asegurar el desempeño de las metas organizaciones.

Las pruebas de significancia de $\beta_{2}(\mathrm{P}>\mathrm{t}: 0,06)$ y $\beta 3$ ( $\left.\mathrm{P}>\mathrm{t}: 0,689\right)$, correspondientes a la unidad de control y cumplimiento y auditoría interna, tampoco muestran relación de significancia con el IDESEMPEST, así que se rechazan las hipótesis $\mathrm{H} 2$ y $\mathrm{H} 3$.

A pesar de que la alta administración y la auditoría interna funcionan de manera alineada, y la unidad de control y conformidad se correlacionan con el desempeño de las estatales, la responsabilidad que el modelo del IAI (2013) atribuye a estas líneas para evaluar la ejecución de los objetivos y de los indicadores, las líneas de defensa tienen bajo poder explicativo sobre el desempeño obtenido por las estatales brasileñas. Esto podría sugerir que el riesgo de la existencia de un desempeño ilegítimo en las estatales es alto.

\section{Interacción entre las líneas de defensa y el desempeño}

Se recurrió al uso del análisis de contenido por la necesidad de asegurar el rigor de la investigación, puesto que los resultados del análisis cuantitativo con el abordaje de los informes evidenciaron que las líneas de defensa tienen bajo poder de explicación sobre el desempeño de las estatales.

Se seleccionaron apartados de los informes de administración, referentes a la gestión de riesgos, controles internos y conformidad y de la auditoría interna para profundizar en los motivos por los cuales la segunda línea de defensa no mostraba relación con la primera y la tercera línea en el análisis de la correlación de Spearman. El 66\% del contenido de los informes fue considerado por el software, en detrimento del 19\% que fue descartado, por contener términos que no estaban alineados a la intención de sentido de las clases 
originadas en la clasificación jerárquica descendente. El análisis de la intención de sentido dio origen a tres clases, que agruparon las palabras que poseían la misma intención de sentido, por medio del p-valor atribuido a cada una de esas palabras por el Iramuteq ${ }^{\circ}$.

Las palabras que presentaron los mayores niveles de significancia fueron agrupadas en la clase 1, plan ( $\mathrm{p}$ $<0,0001)$, deficiencia ( $\mathrm{p}<0,00013)$ y administración $(\mathrm{p}<0,00017)$. Este resultado indica que parte de los informes de administración presentan las funciones de la alta administración como primera línea de defensa, especialmente en el reporte de deficiencias al ejecutar los planes, como evidencia el siguiente pasaje: "en el ámbito del programa de intervención, ocurre la revisión [...] de las deficiencias identificadas en el monitoreo de los plazos [...] para implementación del plan de acción”.

Las palabras agrupadas en la clase 2 que presentaron mayores niveles de significancia consistieron en riesgo ( $\mathrm{p}<0,0001)$, política $(\mathrm{p}<0,0001)$, empresa $(\mathrm{p}<0,00016)$ y riesgos $(\mathrm{p}<0,00019)$. La agrupación de estas palabras muestra que parte de los informes de administración de las organizaciones también evidenció las funciones desempeñadas por las unidades de control y conformidad de las estatales, incluso aseguradas por la alta administración, al señalar la existencia de políticas de gestión de riesgos conforme a este fragmento: "El proceso de gestión de riesgos de las empresas [...] se rige por una política de gestión de riesgos, corporativa y única, aprobada por su Consejo de Administración".

La clase 2 agrupó la mayor cantidad de palabras, con los mayores niveles de significancia en los textos. Este resultado apunta que las funciones de las unidades de control y conformidad, fueron más evidenciadas por la alta administración de las estatales. Este resultado fue contra los niveles de significancia de la segunda línea de defensa en el análisis de la correlación. Además, el análisis del contexto del contenido evidenció que existe relación entre la primera y segunda línea de defensa, no evidenciada por el análisis cuantitativo. Esto refuerza la obtención de un probable desempeño ilegítimo por las estatales, ya que el contenido de los informes difiere de lo que se ha señalado como relación entre los índices.

Las palabras agrupadas en la clase 3 que presentaron los mayores niveles de significancia fueron auditoría interna ( $p<0,0001)$, unidad ( $p<0,0001)$ y ejercicio $(p<0,0001)$. La agrupación de estas palabras apuntó que otra parte de los informes de administración evidenciaron funciones de la auditoría interna, conforme corrobora el siguiente pasaje: "la Auditoría Interna está subordinada al Consejo de Administración y promueve el examen de las actividades desarrolladas por las unidades organizativas con el objetivo de analizar la gestión de las mismas".

La intención de sentido de cada clase se relacionó con una función de las tres líneas de defensa del modelo del IAI (2013). El análisis factorial entre estas clases comprobó que fueron evidenciadas en la mayor parte de los informes, pero sin la existencia de procedimientos estandarizados, conforme presenta la figura 1. 


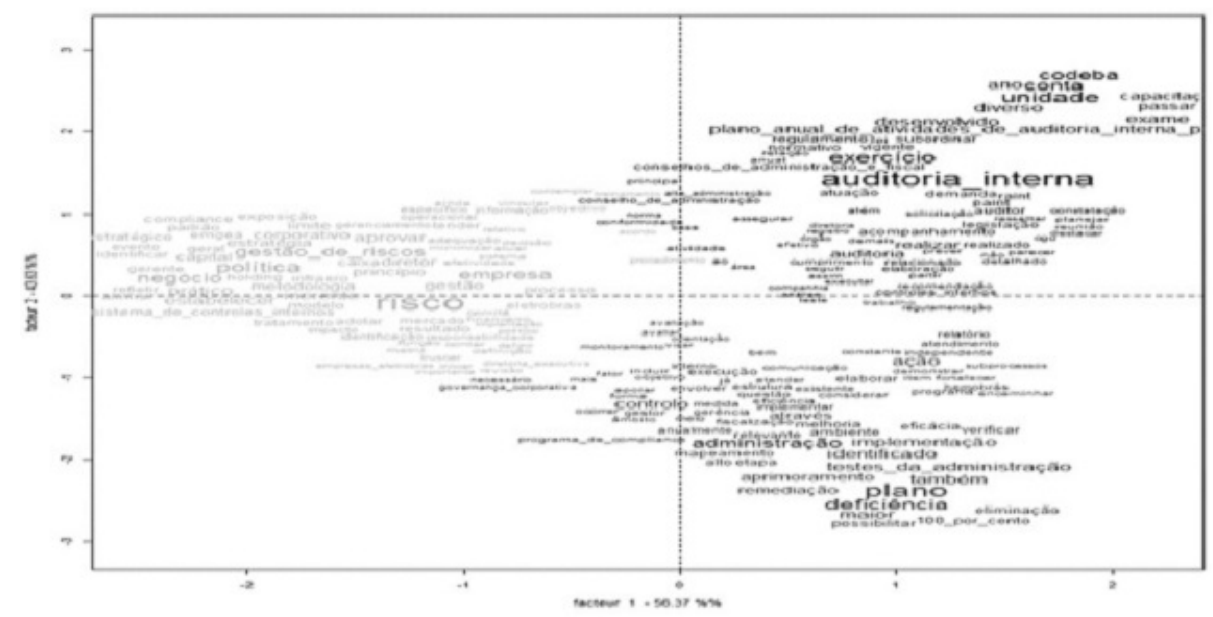

FIGURA 1

Análisis factorial de las clases de sentidos que corresponden a las Tres Líneas de Defensa Fuente: análisis factorial desarrollado por el Iramuteq ${ }^{\circ}$ con base en el corpus textual preparado con informes de administración, de gestión y de auditoría interna (2016).

Gráficamente, el análisis factorial de las clases de sentido presentó cierta simetría en la distribución de las palabras en el transcurso del contenido de todos los informes, en que el 56,37\% de las palabras de los informes de administración se concentraron en el eje de las abscisas, mientras que el 43,63\% se concentraron en el eje de las coordenadas (figura 1).

Las tres clases presentaron una misma extensión de uso de las palabras en los textos, lo que sostuvo el hecho de los informes de las estatales, a pesar de no presentar un patrón en la forma de divulgación de la información, evidencian un contenido parecido con relación a la actuación de la alta administración, unidad de control y cumplimiento y auditoría interna. Esto se explica porque las palabras con mayor nivel de significancia no presentan un alto nivel de dispersión y corrobora los resultados obtenidos por el análisis cuantitativo, en cuanto al funcionamiento de las tres líneas de defensa en las estatales.

La figura 1 también muestra que las acciones realizadas por la segunda línea de defensa fueron evidenciadas por las estatales con una mayor extensión, teniendo en cuenta que la intención de sentido correspondiente a la clase 2 estuvo más distribuida en el eje de las abscisas. Esto confirma el resultado obtenido por el análisis de la correlación de Spearman, en cuanto a la mayor responsabilidad de la unidad de control y conformidad sobre la forma en que el desempeño se obtiene en las estatales.

La intersección de los ejes sugiere que todos los informes de las empresas divulgaron informaciones correspondientes a las tres líneas de defensa, pero sin una misma extensión de funcionamiento en las estatales, lo que ratifica el resultado obtenido por el análisis de la regresión.

\section{CONCLUSIÓN}

El objetivo de este estudio fue analizar la relación de la alta administración, las unidades de control y conformidad y la auditoría interna de empresas estatales brasileñas -conocidas como líneas de defensa según la IAI (2013) - con el desempeño de esas organizaciones, por medio del Índice de Desempeño de las Empresas Estatales (IDESEMPEST).

La alta administración, la unidad de control de conformidad y la auditoría interna no presentaron procedimientos estándares institucionalizados en las estatales, a pesar de presentar contenido similar en los informes divulgados por esas tres unidades. Además de esta falta de estandarización, las empresas presentaron cierta tendencia a valorar más las medidas financieras que las no financieras para medir el desempeño global, esto refleja el funcionamiento inadecuado de la gestión de riesgos por las líneas de defensa. 
La unidad de control y conformidad fue la que presentó mayor influencia en el desempeño en las empresas estatales, en detrimento de la alta administración y la auditoría interna, que también deberían ser responsables de asegurar ese proceso. Aunque las estatales investigadas muestran un desempeño sin variaciones discrepantes en relación con otras empresas, la medición de ese desempeño no fue ejecutada por las tres unidades responsables de gestionar los riesgos de esas organizaciones, lo que debilita la manera en que se obtiene el rendimiento, lo que apunta a cierta ilegitimidad en la forma en que se obtuvo el desempeño en las empresas estatales.

A pesar de estar sometidas a las reglas del MPDG, la forma en que las estatales miden el desempeño evidencia cierto distanciamiento entre la gestión de riesgos y la obtención de ese desempeño. La alta administración y la auditoría interna a pesar de estar alineadas, no fueron suficientes para asegurar la obtención del desempeño organizacional, haciendo que esa responsabilidad recaiga más sobre la unidad de control y conformidad.

El funcionamiento desordenado entre la alta administración, la unidad de control y conformidad y la auditoría interna, ponen en "jaque" el funcionamiento del modelo del IAI (2013) en las estatales brasileñas, y el impacto de ello puede ser justificado en el desempeño obtenido por las compañías, porque la segunda línea de defensa parece ser la que más se esfuerza para que las estatales tengan un mejor desempeño.

\section{Recomendaciones}

Entre las limitaciones del estudio se señala la poca cantidad de informes divulgados en los sitios institucionales de las empresas lo que afectó el tamaño la muestra, además de las informaciones de algunos informes divulgados en disconformidad con la propuesta del modelo del IAI (2013). Para futuras investigaciones se recomienda una comparación entre el contenido evidenciado por las estatales de otros países, para hacer más preciso el análisis de la viabilidad del modelo del IAI (2013).

\section{Referencias}

Anderson, D., \& Eubanks, G. (2015). Leveraging COSO across The three lines of defense. Institute Internal of Auditors (IIA). Online https://www.coso.org/Documents /COSO-2015-3LOD.pdf

Agência Nacional de Saúde Suplementar (ANS) (2017). Método de Cálculo IDSS 2015. Online http://www.ans.gov.br/images/stories/Materiais_para_pesquisa /Perfil_setor/idss/ pqo2015_6_metodo_de_calculo_idss_ano_base_2014.pdf

Bank for Internal Settlements (Banco de Pagamentos Internacionais - BIS). The Internal Audit Function in Banks (2012). Online http://www.bis.org/publ/bcbs176.pdf.

Bonacim, C. A., \& Araújo, A. M. (2010). Influência do capital intelectual na avaliação de desempenho aplicada ao setor hospitalar. Ciência \& Saúde Coletiva, 15(1), 1249-1261. http://dx.doi.org/10.1590/ S1413-81232010000700034.

Clemens, D. (2014). Optimized Integrated Assurance. Internal Auditor. Octubre. Online https://iaonline.theiia.org/ optimized-integrated-assurance

DeFond, M., \& Zhang, J. (2014). A review of archival auditing research. Journal of Accounting and Economics, 58(2-3), 275-326. https://doi.org/10.1016/j.jacceco.2014.09.002

Doyle, J., Ge, W., \& McVay, S. (2007). Determinants of weaknesses in internal control over financial reporting. Journal of accounting and Economics, 44(1-2), 193-223. https://doi.org/10.1016/j.jacceco.2006.10.003

Doughty, K. (2011). The three lines of defence related to risk governance. Isaca Journal, 5. Online https:// www.isaca.org/Journal/archives/2011/Volume-5/Pages/default.aspx

Girão, L. F. (2016). Competição por informações, ciclo de vida e custo do capital no Brasil. Tese de Doutorado em Contabilidade. Universidade Federal Da Paraíba, João Pessoa, PB, Brasil. 
Institute Internal of Auditors (IAI). (2013) The three lines of defence in effective risk management and control. Online https://na.theiia.org/standards-guidance/Public\%20Documents/PP\%20The\%20Three \%20Lines\%20of\%20Defense\%20in\%20Effective\%20Risk\%20Management\%20and\%20Control.pdf

Kaplan, R. S., \& Norton, D. P. (1997). A estratégia em ação: Balanced Scorecard, 11 ed. Editora Campus.

KPMG Auditores Independentes. (2016). KPMG Internal Audit: Top 10 key risks in 2016. Banking, Insurance Companies and Capital Markets.

Kausar, A., Shroff, N., \& White, H. (2016). Real effects of the audit choice. Journal of Accounting and Economics, 62(1), 157-181. https://doi.org/10.1016/j.jacceco.2015. 10.001

Linczuk, L. M. (2012). Governança aplicada a administração pública - a contribuição da auditoria interna para sua efetivação: um estudo em universidades públicas federais. Dissertação. Universidade Tecnológica Federal do Paraná (UTFPR). Curitiba, Brasil.

Lunkes, R. J., Gasparetto, V., \& Schnorrenberger, D. (2011). Um estudo sobre as funções da controladoria. Revista de Contabilidade e Organizações (RCO), 4(10), 106-126. https://doi.org/10.11606/rco.v4i10.34779

Luke, B., Barraket, J., \& Eversole, R. (2013). Measurement as legitimacy versus legitimacy of measures Performance evaluation of social enterprise. Qualitative Research in Accounting \& Management, 10(3/4), 234-258. DOI: 10.1108/QRAM-08-2012-0034

Marks, N. (2016). Some authoritative guidance on risk management and the three lines of defense. https://normanmarks.wordpress.com/2016/03/19/some-authoritative-guidance-on-riskmanagement-and-the-three-lines-of-defense/

Martin, N. C., Santos, L. R. D., \& Dias Filho, J. M. (2004). Governança empresarial, riscos e controles internos: a emergência de um novo modelo de controladoria. Revista Contabilidade \& Finanças, 15(34), 07-22. http:// dx.doi.org/10.1590/S1519-70772004000100001

Ministério do Planejamento, Desenvolvimento e Gestão (MPDG). (2016). Perfil das Empresas Estatais Federais 2015Ano-Base 2014. Instituto Brasileiro de Geografia e Estatística (IBGE). Brasília: MP/SE/DEST.

Mont, J. (2015). What critics say on three lines of defense strategy. Accounting and Auditing. https:// www.complianceweek.com/. Abril/2017

Müller, C. J. (2003). Modelagem de gestão integrando planejamento estratégico, sistemas de avaliação de desempenho e gerenciamento de processos (Modelo de Estratégia, Indicadores e Operaçôes - MEIO). Tese. Universidade Federal do Rio Grande do Sul (UFRGS). Porto Alegre, Brasil.

Porta, F. C. (2011). As diferenças entre Auditoria Interna e Compliance. Dissertação. Universidade Federal do Rio Grande do Sul (UFRGS). Porto Alegre/ Brasil.

Quadros, J. J. (2015). As Contribuições da Auditoria Interna com a Aplicação da Metodologia de Dimensões Organizacionais: Estudo Realizado em uma Instituição Financeira - Um Estudo de Caso. Dissertação. Instituto Superior de Administração e Economia (ISAE-PR). Curitiba/Brasil.

Seago, J. (2015). Risk Management Defense. Internal Auditor. Outubro/2017.

Tabuena, J. (2015). Applying the three lines of defense model. Compliance Week Feb. Academic OneFile.

\section{Notas}

* Artículo de investigación científica.

Licencia Creative Commons CC BY 4.0 